\title{
An Industry Perspective: Future of Anti-Amyloid Trials
}

\section{L.D. Kramer}

Correspondance author: Lynn D. Kramer, MD, Eisai Inc. Woodcliff Lake New Jersey, 07677, USA, Email: lynn_kramer@eisai.com, Telephone: 201-949-4342

Trial outcomes across a spectrum of investigational therapies targeting the "amyloid cascade" for modifying the course of Alzheimer's disease have generated controversy and cast doubts about the potential for success of the amyloid pathway as a target. The use of the term "amyloid cascade" as a singular molecular target is misleading. Given the differentiated molecular and cellular mechanisms interacting in amyloid- $\beta$ homeostasis and the non-linear, dynamic nature of the biochemical process, the terms "amyloid pathway" or "amyloid cycle" have been proposed.

Anti-amyloid compounds have targeted different steps of the cascade including: a) blocking cleavage of the precursor protein at the cell membrane level, thus reducing the production of amyloid- $\beta$ fragments ( $\beta$ - and gamma $(\gamma)$ - secretase inhibitors); b) enhancing clearance mechanisms hoping to alter the dynamic of the many soluble amyloid- $\beta$ species leading to amyloid plaque formation and deposition; and c) clearing plaque itself with therapeutic antibodies. The initial designs and goals of these studies varied from small proof of concept biomarker targeted trials (many of which have efficacy endpoints embedded), safety trials with efficacy endpoint analysis, and large efficacy trials. The reasons for "failure" or the perception of failure differ among trials as well as the therapeutic targets within the "cascade" (1).

To quote an old expression, "let us not throw the baby out with the bathwater" before carefully examining the situation. A critical assessment of the various putative therapeutics trials is imperative for a full comprehension of the results. Once more completely understood, these data tell a remarkable story. As Aisen and colleagues (2) observed, the results of these trials are not singular "failures," and several recently completed trials with more extensive data suggest a potentially different story. The evolving overall story may now actually be gaining in clarity.

For a therapy to be effective, you must have the right hypothesis, the right target within that hypothesis, the right population, the right drug at the right dose with the right treatment duration, and the right trial design for addressing the study objectives. Missing any one of these critical aspects can lead to failure with negative study outcomes. The pathway to future drug development lies in precision pharmacology and medicine (4). For example, sequential biomarker-guided (3) designs or studies, formulating and testing of generated (amyloidrelated) hypotheses is an efficient way to make trials and medicine more effective and precise $(1,4)$. Evaluation of the trial outcomes requires the right interpretation of the results to obtain the right conclusion. Of course, unexpected safety issues can lead to failure because of trial stoppage or dosage adjustment that impact the trial outcome. The "failures" to date are likely attributable to missing on one or more of these steps and they reinforce the need to consider the learnings from these "failures" when developing and interpreting new Alzheimer's clinical trials.

Some investigational compounds have had the wrong target in the amyloid pathway or have had safety issues $(1,3)$. Some trials have been too small or of too short duration to expect differences in cognitive endpoints, despite showing relevant biomarker changes $(1,3)$, whereas other studies demonstrated dose-dependent responses but had suboptimal outcomes due to insufficient dosing $(1,3)$. Moreover, post-hoc analyses have often shown that additional study methodology and design factors, such as time of exposure to higher doses and genetic background (i.e. APOE and sex), can all influence the final results.

In developing therapies for various medical conditions, it is rare that each step in a chemical pathway involved in the condition has the same potential to convey a benefit as a therapeutic target and so we must continue to strive for a deeper understanding of the pathway to help elucidate the best potential intervention strategies. As the task force highlights, we have gained a "better understanding of the specific amyloid species targeted by different antibodies, advancing our insight into the mechanism by which those antibodies may reduce pathology, implementing more comprehensive repertoires of biomarkers into trials, and identifying appropriate doses (5)."

It is well established that biomarker-based trials are essential to optimize go / no-go decisions and reduce friction through the development phases of the trial $(1,3)$. Disease specific biomarkers have the potential to inform target engagement protocols as well as patient selection, efficacy, and safety monitoring. For 
instance, biomarkers can help inform the development of a new generation $\beta$-secretase 1 (BACE1) inhibitors with high substrate selectivity for the amyloid precursor protein, thus minimizing the risk of some of the synaptic off-target effects resulting in key safety observations (3). Furthermore, novel biomarkers tracking neuroinflammatory processes are needed to efficiently investigate the role of immune modulation in amyloid therapeutic clearance.

In conclusion, biomarkers will support the development of pathway (mechanism)-based therapies for suitable target populations (precision pharmacology) that will enrich the holistic, individualized prevention and management of neurological diseases, such as Alzheimer's disease, as already successfully demonstrated in drug development in the field of oncology (4).
Disclosure: Dr. Kramer is an employee of Eisai Inc.

\section{References}

1. Aisen PS, Vellas B, Hampel H. Moving towards early clinical trials for amyloid-targeted therapy in Alzheimer's disease. Nat Rev Drug Discov. 2013;12(4):324.

2. P.S. Aisen, J. Cummings, R. Doody, L. Kramer, S. Salloway, D.J. Selkoe, J. Sims, R.A. Sperling, B. Vellas and the EU/US CTAD 2019 Task Force*. The Future of Anti-Amyloid Trials. J Prev Alz Dis 2020;3(7):146-151.

3. Hampel H, Frank R, Broich K, et al. Biomarkers for Alzheimer's disease: academic, industry and regulatory perspectives. Nat Rev Drug Discov. 2010;9(7):560-574.

4. Hampel H, Vergallo A, Aguilar LF, et al. Precision pharmacology for Alzheimer's disease. Pharmacol Res. 2018;130:331-365.

5. van Dyck CH. Anti-Amyloid- $\beta$ Monoclonal Antibodies for Alzheimer's Disease: Pitfalls and Promise. Biol Psychiatry. 2018;83(4):311-319.

6. Hampel H, Vassar R, De Strooper B, et al. The $\beta$-Secretase BACE1 in Alzheimer's Disease. Biol Psychiatry. 2020; published online: doi:10.1016/j. biopsych.2020.02.001 